\title{
Association of Occupation and Breast Cancer Mortality in the State of Vermont, 1989-1993
}

\author{
Dominic F. Geffken*, M.D., Melissa J. Perry ${ }^{\dagger}$, Sc.D., and \\ Peter W. Callas ${ }^{\S}$ Ph.D.
}

\begin{abstract}
Vermont's breast cancer death rate is among the highest in the U.S. This study analyzed the association between breast cancer mortality and occupation in Vermont women. Given that Vermont is a rural state, one initial hypothesis was that occupational exposure to pesticides might partly explain the high death rate. Death certificate data from 1989-1993 were analyzed to determine relative risk of breast cancer death according to occupation. Case-control analysis demonstrated increased relative risk of breast cancer death for women in two broad occupational groups: 1) Executive, Administrator and Managers and 2) Professionals. Decreased relative risk of breast cancer death was seen for the occupational group of Homemaker. Data indicated that women in the occupational group of Farming, Forestry, and Fishing were not at increased risk of dying from breast cancer. The associations of occupation and breast cancer mortality in Vermont women do not differ significantly from those seen in larger U.S. studies.
\end{abstract}

\section{INTRODUCTION}

Vermont's breast cancer death rate is among the highest of U.S. states at approximately 29 deaths per 100,000 women. Each year nearly 100 Vermont women die from breast cancer and between 200 - 400 women are diagnosed with the disease $(1,2)$. Breast cancer is the second leading cause of cancer mortality in American women (3). The incidence of breast cancer is increased in populations of women that are more affluent when compared to less wealthy populations. The rate of breast cancer mortality is 20.9 per 100,000 women in the United States, compared to nations such as Mexico and

\footnotetext{
* To whom correspondence should be addressed: Family Medicine Residency Program, Department of Family Medicine and Community Health, University of Massachusetts, University Campus, 55 Lake Avenue North, Worcester, MA, 01655, USA. E-mail: Dominic.Geffken@vtmednet.org.

$\dagger$ Occupational Health Program, Harvard School of Public Health, Boston, MA 02115.

$\S$ University of Vermont, College of Medicine, Burlington, VT 05405 .
}

China where the mortality rates are 8.9 and 6.2 per 100,000, respectively (4).

Previous studies have demonstrated that women in professional occupational groups are at increased risk for breast cancer mortality $(5,6)$. This is likely due to social and economic factors associated with the disease. Risk factors identified as predisposing women for developing breast cancer include high socioeconomic status (SES) $(7,8)$, early age of menarche $(9,10)$, nulliparity and advanced age of first birth (8). In addition, exposure to certain chemicals, such as pesticides used in agriculture, have been shown to be associated with developing breast cancer (11-14). Vermont is a rural state in New England and one purpose of this study was to look at the association of breast cancer with occupation in this setting.

In trying to draw this association, steps were taken to control for the "healthy worker effect" (15). The healthy worker effect describes the epidemiological finding that employed persons have lower mortality rates compared to the general population because continued 
employment depends on good health. Certain occupational groups may have an increase in a specific cause of death (in this case breast cancer) due to a decrease in other causes of death (e.g. ischemic heart disease) because they are healthier than the general population or than other occupational groups. The prevalence of ischemic heart disease has been shown to exhibit a healthy worker effect (16). In our analyses we constructed a control group that excluded women who died of ischemic heart disease and are therefore likely to be "less healthy", in an attempt to control for the healthy worker effect.

In this study, data pertaining to occupation and cause of death were obtained from death certificates. Death certificate data are important sources of preliminary information about causes of mortality in a state or locale. Despite well known limitations such as the great variability in the accuracy of occupations reported in death certificates (17-19) these data are attractive to use because of their ready availability, uniformity between counties, and lack of expense. In states where breast cancer incidence data are not centralized or where mammography registries are in their infancy, death certificate data represent information that can be used initially to identify populations at risk and to develop stronger hypotheses that can be answered with subsequent incidence data when available.

\section{METHODS}

Death certificates inclusive for the years 1989 - 1993 were obtained from the Vermont Health Department. A total of 12,765 female deaths were recorded for the entire state of Vermont during the five year period, of which 512 had breast cancer identified as the underlying cause of death. Data obtained from the death certificates were underlying cause of death, occupation, race, and age. No personal identifiers were used in this study.

Preliminary descriptive statistics were performed by analyzing the distribution of age and race of the women who died from breast cancer within this population. A series of case-control analyses were then performed. Case subjects were defined as all women for whom breast cancer was recorded as the underlying cause of death $(n=512)$. Three separate control groups were created from the 12,253 women who died from causes other than breast cancer. The control groups were created as follows: in case-control analysis 1 , the control group was selected from all non-breast cancer deaths; in case-control analysis 2 , the control group was selected from all deaths except breast cancer and female reproductive system malignancies (e.g. ovarian or cervical malignancies); and in case-control analysis 3, the control group was composed of "healthy workers" in which deaths due to ischemic heart disease (ICD-9 codes, 410-414) (20) and deaths due to breast cancer were excluded. We constructed these three different control groups to elucidate the etiology of any significant risk we observed. If for example we observed a decreased risk of breast cancer death in Occupation $\mathrm{A}$ for case-control analysis 1 but an increased risk for casecontrol analysis 2 we might hypothesize that the odds ratio (OR) was underestimated in the first due to the inclusion of female reproductive system controls, which may have risk factors similar to those of breast cancer. Alternatively, if we observed a decreased risk of breast cancer death in Occupation A for case-control analysis 1 but an increased risk in case-control analysis 3 this would suggest that Occupation A had a large number of "unhealthy" women who were dying from causes other than breast cancer.

Controls were matched to cases by age ( \pm 1 year). For each control group, three controls per case were randomly selected from a pool of potential controls, resulting in 1536 controls per group.

Mantel-Haenszel odds ratios (21) and corresponding $95 \%$ confidence intervals (CI) were computed controlling for age, to estimate the relative risk for 16 broad occupational categories. Because this is a casecontrol study the relative risk cannot be directly calculated. The Mantel-Haenszel method was used because breast cancer death is relatively rare event and the OR can be used to estimate the relative risk, which is the breast cancer death rate in a particular occupational category divided by the breast cancer death rate for women not in that occupational category. Decedent occupational data were coded according to the 1980 Bureau of the Census classification system also known as the Standard Occupational Classification (SOC) System. The division of occupation in the SOC system was established for the 1980 U.S. Census as a standard to compare occupational data obtained from household surveys with occupational data obtained from industry. Prior to this attempt to standardize occupational categories, the Bureau of the Census and industry groups had separate systems that were not easily comparable (22).

\section{RESULTS}

In this study, the mean age at death for the breast cancer cases was 67.5 years, compared to an average age of 77.5 years for women dying from other causes. Analysis of breast cancer deaths by race showed that 99.6\% occurred in Caucasian females which is consistent with the racial distribution in Vermont.

Risk analyses demonstrated that Vermont women in the broad occupational groups of 1) Executive, Administrative, and Managerial occupations and 2) Professional occupations were at higher risk from dying 
of breast cancer than other occupational groups (Table 1). Housewives showed a decreased risk of dying from breast cancer in comparison to other occupational groups. Risk analyses also demonstrated that Vermont women in the Farming, Forestry and Fishing occupational group had a non-significant decrease in risk of dying from breast cancer although only one breast cancer death was recorded for that group. All other occupations showed no significant increased or decreased risk of dying from breast cancer, although again the number of deaths were small for some occupations. There was no evidence of a "healthy worker effect" in any of the occupational groups. The same trends in occupational risk were observed in all three of the case-control analyses. For example, for the three case-control groups in the Executive, Administrative and Managerial Occupational Group the following results were obtained: case-control analysis $1, \mathrm{OR}=1.61,95 \% \mathrm{CI}=1.09-2.28$; case-control analysis $2, \mathrm{OR}=1.55,95 \% \mathrm{CI}=1.05-2.28$; case-control analysis $3, \mathrm{OR}=1.48,95 \% \mathrm{CI}=1.01-2.18$. The three case-control analyses for the Homemaker Occupational Group produced the following results: case-control analysis $1, \mathrm{OR}=0.66,95 \% \mathrm{CI}=0.53-0.83$; case-control analysis $2, \mathrm{OR}=0.65,95 \% \mathrm{CI}=0.52-0.82$; case-control analysis $3, \mathrm{OR}=0.69,95 \% \mathrm{CI}=0.55-0.86$. For ease of interpretation only results of case-control analysis 1 (breast cancer deaths vs. non-breast cancer deaths) are shown in Table 1.

\section{DISCUSSION}

The major findings of this case-control analysis are: 1) there was an increased relative risk of breast cancer death in two broad occupational groups A) Executives, Administrators and Managers and B) Professionals; 2) there was a decreased relative risk of breast cancer death seen in the occupational group of Homemaker; and 3) there was a non-significant decrease in relative risk of breast cancer mortality in women in the occupational group of Farming, Forestry or Fishing.

The association of occupation with breast cancer mortality is not remarkably different in Vermont women when compared with the results of larger U.S. studies. In our study we found that occupational groups at increased risk of dying form breast cancer were Executives, including Administrators and Managers (total number of deaths from all causes $n=590$ ) and Professionals (total number of deaths from all causes $n=1768)$. These results are similar to previous studies (6).

The women in the two occupational groups that exhibited increased risk of dying of breast cancer have no obvious predisposing workplace exposure that would explain this increased risk. However, women in these occupational groups are likely to be at increased risk due to lifestyle factors that affect reproduction and estrogen exposure, such as SES, parity and maternal age at first birth.

High SES has been identified as a positive risk factor for the development of breast cancer $(7,8)$ and it is also associated with numerous other positive risk factors. Data for the Burlington Vermont Metropolitan Area indicate that the range of mean weekly earnings for Administrative and Professional occupations was \$521 - \$905 and \$594 - \$881, respectively (23). This was compared with the mean weekly wage of $\$ 305-\$ 464$ for clerical workers, a group that demonstrated no significant increased or decreased risk for breast cancer in our study. Women who enter occupations with higher monetary compensation are themselves more likely to come from higher SES backgrounds $(24,25)$. Menarche has been shown to occur at a younger age for high SES females when compared to lower SES females (26) and other studies have found early age of menarche to be a risk factor for developing breast cancer $(9,10)$.

Women in high SES groups may also have children at a later age. Vermont data indicate that a greater number of older women are experiencing the birth of their first child. In 1990, 24\% of first births occurred among women over 30 years old (an age that is considered advanced maternal age) compared to women 19 years old and under, who accounted for only $16.5 \%$ of the first births (27). According to national U.S. data, 33\% of the births were first births for women 30-44 years of age in the Executive, Administrative, Managerial and Professional Occupational Groups while only $9 \%$ of the births were first births for Service sector women in the same age group (28). Advanced maternal age at time of first birth has been identified as a risk factor for developing breast cancer (8).

Compared to the professional occupational groups the women in the Homemaker occupational group may have had children at an earlier average age. The present study found that women in the occupational group of homemaker (total number of deaths from all causes $n=$ 5143) were at decreased risk of dying from breast cancer. These findings are similar to a much larger study in which 2.9 million U.S. death certificates were analyzed using the National Institute for Occupational Safety and Health (NIOSH) data set (6).

There is one discrepancy in the findings of our study compared to the national study (6) that found the occupational group of Administrative Support Including Clerical workers to be at increased risk of death from breast cancer. This was not seen in the Vermont sample. The difference may be attributable to the difference in size of the two study populations with the number of women in the administrative support population being 1414 in our study, compared to 111,421 women in the Rubin et al. study (6). 
Table 1. Mantel-Haenszel odds ratios and 95\% confidence intervals (CI) of breast cancer death by 16 broad occupational categories for casecontrol analyses.

\begin{tabular}{|c|c|c|c|c|}
\hline Broad Occupational Groups & $\begin{array}{l}\text { Cause of Death: } \\
\text { Breast Cancer } \\
\quad(n=512)\end{array}$ & $\begin{array}{c}\text { Cause of Death: } \\
\text { Non-Breast Cancer } \\
(n=1536)\end{array}$ & Odds Ratio ${ }^{a}$ & $\mathbf{9 5 \%} \mathbf{C I}$ \\
\hline Executive, Administrative, Managerial & 41 & 79 & 1.61 & $1.09-2.38$ \\
\hline Professional & 83 & 183 & 1.44 & $1.08-1.91$ \\
\hline Technicians and Related Support Occupations & 12 & 33 & 1.10 & $0.56-2.13$ \\
\hline Sales & 34 & 107 & 0.95 & $0.64-1.42$ \\
\hline Administrative Support, Including Clerical & 73 & 184 & 1.22 & $0.91-1.63$ \\
\hline Service & 53 & 177 & 0.89 & $0.64-1.23$ \\
\hline Farming, Forestry, and Fishing & 1 & 17 & 0.17 & $0.03-1.04$ \\
\hline Mechanics and Repairers & 1 & 2 & 1.48 & $0.15-15.09$ \\
\hline Construction & 0 & 6 & - & - \\
\hline Precision Production & 9 & 22 & 1.23 & $0.56-2.66$ \\
\hline Machine Operators and Assemblers & 35 & 87 & 1.22 & $0.81-1.84$ \\
\hline Transportation & 1 & 5 & 0.62 & $0.07-5.12$ \\
\hline Equipment Cleaners, Laborers, and Helpers & 10 & 30 & 1.00 & $0.49-2.05$ \\
\hline Armed Forces & 2 & 0 & - & - \\
\hline Homemakers & 147 & 576 & 0.66 & $0.53-0.83$ \\
\hline Never Worked & 6 & 19 & 0.94 & $0.38-2.32$ \\
\hline
\end{tabular}

a Adjusted for age using Mantel-Haenszel analysis.

The relatively small size of the population we studied contributed to our findings of very few breast cancer deaths in several occupational groups. This was evident in the analysis of the Farming, Forestry, or Fishing occupational group, which showed a non-significant decrease in relative risk of breast cancer mortality, though there was only one breast cancer death in the case control analysis. One of our initial hypotheses was that Vermont women had a high rate of breast cancer mortality because more women were involved in agriculture and were exposed to pesticides. Our results do not support this hypothesis though the small size of this occupational group prevents us from making strong claims. In any case, employment in an agricultural occupation may not be a good predictor of pesticide exposure. A study of Swedish female farmers demonstrated that they were at decreased risk of developing breast cancer (29). A recent study showed organochlorine tissue levels were associated with breast cancer in women from the New York City area (14), suggesting that pesticide residue may be a better predictor of breast cancer development in urban or rural women rather than actual occupation. Future studies of the association of breast cancer and pesticide exposure in Vermont women should use serum measurements of organochlorines to determine pesticide exposure rather than occupation as a proxy measure.

There was no evidence of the "healthy worker effect" in any of the occupational groups analyzed. Specifically, there were no changes in statistical significance or nonsignificance when the "healthy worker" case-control groups (case-control analysis 3) were compared to the other case-control analyses. Since the relative risk was similar in all of the case-control analyses conducted, it is unlikely that the increase in breast cancer risk was due to one occupational group or a subset of one occupational group being healthier than another.

Among the limitations of this study is the use of death certificates to gain information about the occupations of study subjects. It has been observed that there is great variability in the accuracy of the occupations reported in death certificates (18). One study comparing occupational data reported on death certificates with that obtained from personal histories of surviving family members yielded an overall match rate of $47.9 \%$ (19). However, it is noteworthy that white females, the group that made up our study population almost exclusively, had the highest percentage of exact matches for usual occupation at $60 \%$ (19). To our knowledge there have been no studies looking at the accuracy of data retrieved from Vermont death certificates. Death certificate data remain important sources of preliminary information about causes of mortality in a state or locale because of their ready availability, uniformity between counties, and lack of expense. Identified populations at increased risk for breast cancer mortality may benefit from increased surveillance and further etiologic research into the factors that place them at increased risk.

The reasons for Vermont's high breast cancer mortality are still unknown. In terms of occupational risk, data from Vermont do not differ greatly from the rest of the U.S. It is unclear at the present time if Vermont's high breast cancer mortality is due to 
increased incidence or due to later stage of detection which reduces survival. A state-wide mammography registry was initiated in 1994. These data should become available for analysis in the next 1-2 years and will be instrumental in determining the origins of Vermont's high breast cancer mortality and for indicating which populations should be targeted for more aggressive educational and preventive services. Until then, careful monitoring of state-level mortality data provide important demographic indicators of excess mortality that warrant further attention.

\section{ACKNOWLEDGMENTS}

The authors would like to thank the Dean's Office, the Department of Family Practice, and the Office of Health Promotion at the University of Vermont College of Medicine for funding this project. We would also like to thank Mr. Rob McIntyre at the Vermont Health Department for supplying the data for this study.

\section{REFERENCES}

1. American Cancer Society. Cancer Facts and Figures. Atlanta, GA; 1995

2. Miller B, Ries L, Hankey B, et al. SEER Cancer Statistics Review: 1973-1991. Bethesda National Cancer Institute; 1993.

3. Parker S, Tong T, Bolden S, Wingo P. Cancer Statistics, 1997. CA: A Cancer Journal for Clinicians 47: 5-27; 1997.

4. Mettlin C. Global breast cancer mortality statistics. CA: A Cancer Journal for Clinicians 49: 138-144; 1999.

5. Calle EE, Murphy TK, Rodriguez C, et al. Occupation and breast cancer mortality in a prospective cohort of US women. American Journal of Epidemiology 148: 191-197; 1998.

6. Rubin CH, Burnett CA, Halperin WE, Seligman PJ. Occupation as a risk identifier for breast cancer. American Journal of Public Health 83: 1311-1315; 1993.

7. Heck KE, Wagener DK, Schatzkin A, et al. Socioeconomic status and breast cancer mortality, 1989 through 1993: an analysis of education data from death certificates. American Journal of Public Health 87: 1218-1222; 1997.

8. Kelsey JL. Breast cancer epidemiology: summary and future directions. Epidemiological Reviews 15: 256-263; 1993.

9. Peeters PH, Verbeek AL, Krol A, et al. Age at menarche and breast cancer risk in nulliparous women. Breast Cancer Research and Treatment 33: 55-61; 1995.

10. Laing AE, Demenais FM, Williams R, et al. Breast cancer risk factors in African-American women: the Howard University Tumor Registry experience. Journal of the National Medical Association 85: 931-939; 1993.

11. Hoyer AP, Grandjean P, Jorgensen T, et al. Organochlorine exposure and risk of breast cancer. Lancet 352: 1816-1820; 1998.

12. Ardies CM, Dees C. Xenoestrogens significantly enhance risk for breast cancer during growth and adolescence. Medical Hypotheses 50: 457-464; 1998.

13. Guttes S, Failing K, Neumann K, et al. Chlororganic pesticides and polychlorinated biphenyls in breast tissue of women with benign and malignant breast disease. Archives of Environmental Contamination and Toxicology 35: 140-147; 1998.

14. Wolff MS, Toniolo PG, Lee EW, et al. Blood levels of organochlorine residues and risk of breast cancer. Journal of the National Cancer Institute 85: 648-652; 1993.

15. McMichael A, Haynes S, Tyroler H. Observations on the evaluation of occupational data. Journal of Occupational Medicine 17: 128-131; 1975.

16. Reviere R, Eberstein IW. Work, marital status, and heart disease. Health Care for Women International 13: 393-399; 1992.

17. Selikoff IJ. Use of death certificates in epidemiological studies, including occupational hazards: discordance with clinical and autopsy findings. American Journal of Industrial Medicine 22: 469-480; 1992.

18. Mandel J, Schuman L. Epidemiology of cancer of the prostate. In: Lilienfeld A, ed. Reviews of cancer epidemiology. Volume 1. New York: Elsevier; 1980: 1-83.

19. Schade WJ, Swanson GM. Comparison of death certificate occupation and industry data with lifetime occupational histories obtained by interview: variations in the accuracy of death certificate entries. American Journal of Industrial Medicine 14: 121-136; 1988.

20. International Classification of Diseases. 9th Revision, Clinical Modifications (ICD-9-CM). Los Angeles: Practice Management Information Corporation; 1994.

21. Mantel N, Haenszel W. Statistical aspects of the analysis of data from retrospective studies of disease. Journal of the National Cancer Institute 22: 719-748; 1959.

22. U.S. Department of Labor. Revising the Standard Occupational Classification System. Bureau of Labor Statistics Report 929; 1999.

23. U.S. Department of Labor. Occupational compensation survey: Pay only. Burlington Vermont Metropolitan Area. August 1992. Washington, DC: U.S. Government Printing Office, Bureau of Labor Statistics Bulletin: 3065-3060; 1992.

24. Holmes V, Esses L. Factors influencing Canadian high school girls' career motivation. Psychology of Women Quarterly 12: 313-328; 1988.

25. Picou J, Curry E. Structural, interpersonal and behavioral correlates of female adolescents' occupational choices. Adolescence 8: 421-432; 1973.

26. Henneberg M, Louw GJ. Average menarcheal age of higher socioeconomic status urban Cape coloured girls assessed by means of status quo and recall methods. American Journal of Physical Anthropology 96: 1-5; 1995.

27. Vermont Vital Statistics 1990. Burlington, VT: Vermont Department of Health; 1992.

28. U.S. Bureau of Census. Fertility of American women: June 1990. Washington, DC: U.S. Government Printing Office; 1991.

29. Wiklund K, Dich J. Cancer risks among female farmers in Sweden. Cancer Causes and Control 5: 449-457; 1994.

Dominic F. Geffken, MD conducted this research while he was a medical student at the University of Vermont College of Medicine. $\mathrm{He}$ is currently a first year resident in Family Medicine and Preventive Medicine at the University of Massachusetts Medical Center (Worcester, Massachusetts, USA). Melissa Perry, ScD, is currently an instructor in the Occupational Health Program at the Harvard School of Public Health. Peter W. Callas, PhD, is a Research Assistant Professor in Medical Biostatistics at the University of Vermont College of Medicine (Burlington, Vermont, USA). 